\title{
Improved performance of radiochromic films for high-dose dosimetry
}

\author{
S. Akhtar ${ }^{1,2}$, A. Shahzad ${ }^{1 \star}$, S. Bashir ${ }^{3}$, M.Y. Hussain ${ }^{2}$ and N. Akhtar ${ }^{4}$ \\ 1 Department of Physics, Government College University Faisalabad (GCUF), Allama Iqbal Road, Faisalabad 38000, Pakistan. \\ 2 Department of Physics, University of Agriculture Faisalabad (UAF), Jail Road, Faisalabad 38000, Pakistan. \\ 3 Department of Physics, State University of New York (Albany), 1400 Washington Avenue, Albany, NY 12222, USA. \\ ${ }^{4}$ Department of Radiations, Nuclear Institute of Agriculture and Biology (NIAB), Faisalabad 38000, Pakistan.
}

Received 27 February 2015 - Accepted 2 January 2016

\begin{abstract}
Radiochromic thin films based on polyvinyl alcohol (PVA) containing different concentrations of methyl red (MR) and methylene blue (MB) dyes were studied for the quantification of high doses. These films were exposed to doses of 100 to $200 \mathrm{kGy}$. $\mathrm{A}^{137} \mathrm{Cs}$ radiation source was employed for this purpose. The absorption spectra of unirradiated and irradiated dyed PVA films are measured with a UV/VIS spectrophotometer in terms of absorbance at $518 \mathrm{~nm}$ for MR-PVA film and $668 \mathrm{~nm}$ for MB-PVA film. A strong dependence on the dye concentration of the absorbed dose is observed for high-dose irradiation. It was shown that the MR-PVA film is stable before and after irradiation under dark as well as florescent light as compared with MB-PVA film.
\end{abstract}

Keywords: Radiochromic thin films / $\gamma$-rays / irradiation / absorbance / spectrophotometer

\section{Introduction}

The application of radiation processing has been widely expanded around the world. Food preservation and sterilization of medical devices are done with the help of ionizing radiation to keep them safe from microorganisms for a longer time. Since a specific amount of dose delivered is necessary for a specific purpose, an accurate estimate of the dose is necessary. This task of measuring doses is done with the help of dosimeters. For each purpose, there is a different suitable dosimeter used, with specific characteristics.

Radiochromic film dosimeters represent one of the solid types of passive, two-dimensional and weak energy-dependent dosimeters used due to their ruggedness, stability, relatively inexpensive batches and ease of handling. Moreover, the performance of dyed films (bromocresol purple in PVC, nitro-blue tetrazolium in PVB, methyl red in PVB) was also improved by using different solvents for film formation (Alzahrany et al., 2011; Kattan et al., 2011; Rabaeh et al., 2012). An indicator dye-based PVA film containing chloral hydrate has been introduced recently. The color change of the film indicates acid formation, and therefore is very suitable for use as a radiation indicator in some food irradiation applications (Beshir et al., 2013). Radiation-induced decoloration of methyl red in alkaline and acidic aqueous solutions was studied. The absorbance in alkaline medium decreases exponentially with the increase in the absorbed dose for a dose range from 50 to $6000 \mathrm{~Gy}$,

\footnotetext{
^ aamirshahzad_8@hotmail.com
}

while it decreases linearly for doses up to 200 Gy (Ajji et al., 2006).

Several dyed PVA films have been investigated for dosimetric purposes. Nevertheless, PVA films with tetrazolium violet and methyl viologen dyes were introduced for estimating high dosage of gamma radiation (Abdel-Fattah et al., 1999; Lavalle et al., 2007). Cresol red-dyed PVA film blended with trichloroacetic acid irradiated by gamma rays was studied using a UV-Visible spectrophotometer and the Raman spectroscopy method (Susilawati et al., 2009). The absorbance of these dosimeters increases with the absorbed dose up to $50 \mathrm{kGy}$. These dosimeters were not tested beyond a $50 \mathrm{kGy}$ absorbed dose and therefore are not reliable in this dose range. We evaluated the performance of both of our dosimeters up to $200 \mathrm{kGy}$ and obtained a good linear relationship of incident radiation and absorbance.

This work describes the investigation of the dosimetric properties of polyvinyl alcohol-based films with methyl red (MR) or methylene blue (MB) dye at doses up to $200 \mathrm{kGy}$ and compares their relative advantages. The effect of a high concentration of dyes in PVA films as well as pre- and post-irradiation stability on the film response are described. In Section 2, both kinds of PVA films with different dye concentrations were formatted by a casting polymer solution technique. The optical absorption spectra of dyed films were studied in the wavelength range of $200-800 \mathrm{~nm}$ using a UV/VIS spectrophotometer. In Section 3, the results of both the MR-PVA and MB-PVA films are discussed and their 


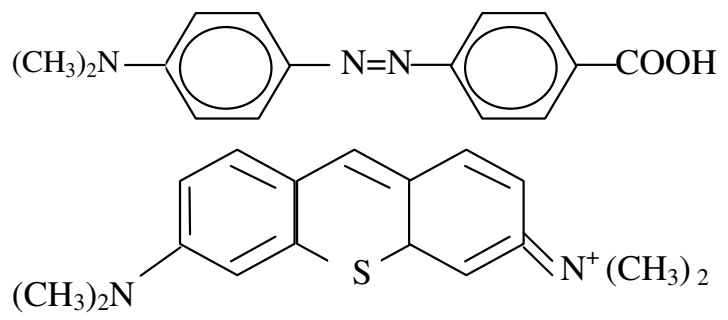

a) Methyl red

b) Methylene blue

Figure 1. Molecular structures of methyl red (MR) and methylene blue (MB).

absorbance characteristics are compared with the published results. The effect of the dye concentration on the performance of the film was also investigated. Finally, this work is summarized in Section 4.

\section{Experimental procedure}

The following materials were used for preparation of the radiochromic films: polyvinyl alcohol (PVA) (VWR International), methyl red (MR) $\left(\mathrm{C}_{15} \mathrm{H}_{15} \mathrm{~N}_{3} \mathrm{O}_{2}, \mathrm{M}: 269.31 \mathrm{~g} \mathrm{~mol}^{-1}\right)$, methylene blue (MB) $\left(\mathrm{C}_{16} \mathrm{H}_{18} \mathrm{~N}_{3} \mathrm{SCl}, \mathrm{M}: 319.9 \mathrm{~g} \mathrm{~mol}^{-1}\right)$ and double-distilled water. The molecular structures of the MR and MB dyes are shown in Figure 1.

The polymer solution was prepared by dissolving $12 \mathrm{~g}$ of polyvinyl alcohol powder in $200 \mathrm{~mL}$ double-distilled water. This PVA solution was stirred at a temperature of $45^{\circ} \mathrm{C}$ for about $24 \mathrm{~h}$ and then left to cool. The PVA solution was divided into four parts. Three different weights of methyl red, 100, 200 and $300 \mathrm{mg}$, were added to three parts of PVA solution, thus making solutions with concentrations of $0.2 \mathrm{~g} \mathrm{~L}^{-1}$, $0.4 \mathrm{~g} \mathrm{~L}^{-1}$ and $0.6 \mathrm{~g} \mathrm{~L}^{-1}$, respectively, while the forth part was kept undyed. To make the solutions homogeneous, all of the four solutions were further stirred for $2 \mathrm{~h}$ at the same temperature. The natural $\mathrm{pH}$ of the MR-PVA solution was 5 (i.e. acidic solution), determined by a Jenway $3510 \mathrm{pH}$ meter. To prepare the PVA thin films, each of the four solutions was poured on a separate horizontal glass plate and left to dry for about $48 \mathrm{~h}$ at room temperature. The red-colored and undyed PVA films were stripped from the glass plates after drying. The average thickness of the film was $33 \pm 3 \mu \mathrm{m}$ with dimensions of $5^{\prime \prime} \times 5^{\prime \prime}$. Each film was wrapped with a black plastic sheet to protect it from environmental factors. The same procedure was repeated to develop blue-colored MB-PVA films; however, these films have a $\mathrm{pH}$ of 6.5 .

The samples were irradiated at the ${ }^{137} \mathrm{Cs}$ gamma source facility of the Nuclear Institute for Agriculture and Biology (NIAB), Faisalabad, Pakistan. The dose rate of the source was measured using a Fricke reference standard dosimeter, and it had a value of $660 \mathrm{~Gy} \mathrm{~h}^{-1}$. A dose in the range of (100200) kGy with a dose step of $10 \mathrm{kGy}$ was delivered to both of the films. The exposure time was estimated from the dose rate and the target dose. The optical absorption spectra of unirradiated and irradiated films were measured with a Perkin Elmer Precisely Lambda 25 UV/VIS spectrophotometer in the wavelength range of (200-800) $\mathrm{nm}$.

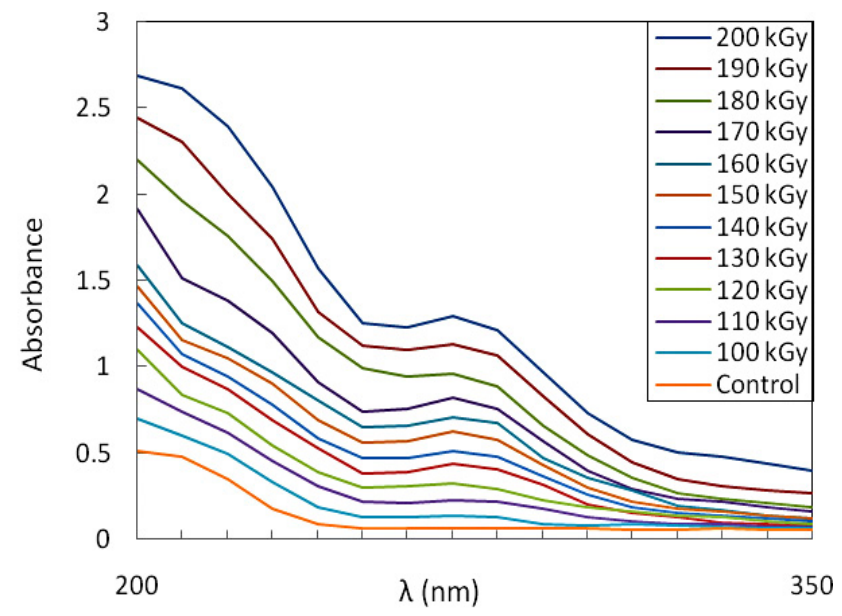

Figure 2. Absorption spectra of unirradiated and irradiated undyed PVA films at different ${ }^{137} \mathrm{Cs} \gamma$-ray doses from $100-200 \mathrm{kGy}$.

\section{Results and discussion}

For the film performance, the effect of dye concentrations with a constant PVA (polymer) amount was tested. At the limit of suitable high doses (100-200 kGy by the ${ }^{137}$ Cs source), the parameters of the dyed film analysis with (dye concentrations and absorbed dose) were determined. The response $\left(\Delta \mathrm{A} / \mathrm{A}_{0}\right)$ calculations obtained for polyvinyl alcohol-based films with methyl red (MR) or methylene blue (MB) dye that we estimated from our experiment, given in Section 2, are presented. The stability of the dyed films under normal laboratory conditions (before and after the influence of irradiation) is discussed. The surprising qualitative results of the dyed polyvinyl alcohol films at the wavelength where absorption was maximum $\left(\lambda_{\max }\right)$ were obtained by using the UV/VIS spectrophotometer with different sets of high concentrations of dyes in PVA solution and choosing higher doses than used earlier, to ensure that the new experimental results of the dyed films are well matched with the earlier known results. It is observed that the absorbance was 0.4 for MR-PVA film and 3.2 for MBPVA at the maximum wavelengths of $518 \mathrm{~nm}$ and $668 \mathrm{~nm}$, respectively.

Figures 2-4 show the main results measured by spectrophotometry for undyed films, dyed films and dye concentration effects, respectively. These figures reveal the comparison of the optical absorbance obtained from the presented method at $\lambda_{\max }$ for dyed films obtained by UV/VIS spectrophotometer of Chung and Miller (1994), Ajji (2006) and Bhat et al. (2007). We found that our results from the presented method are in 


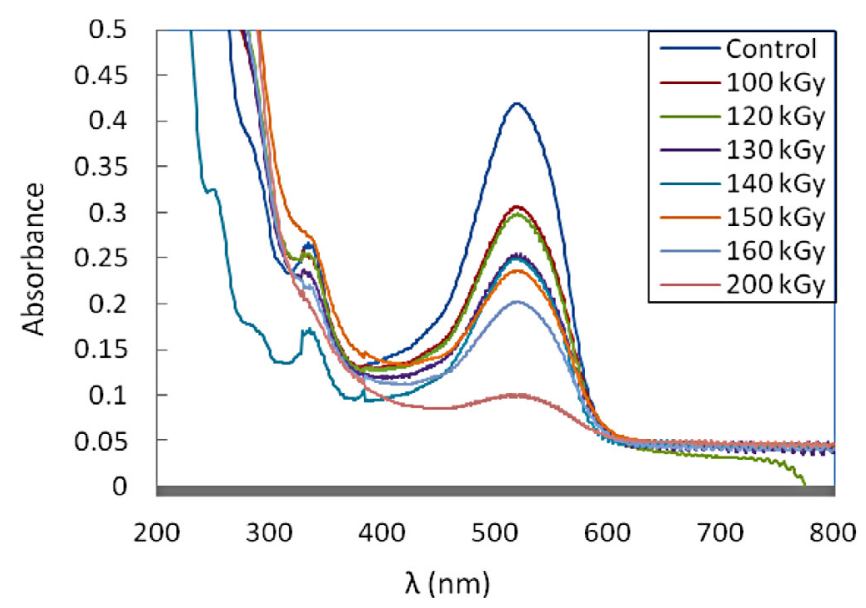

(a)

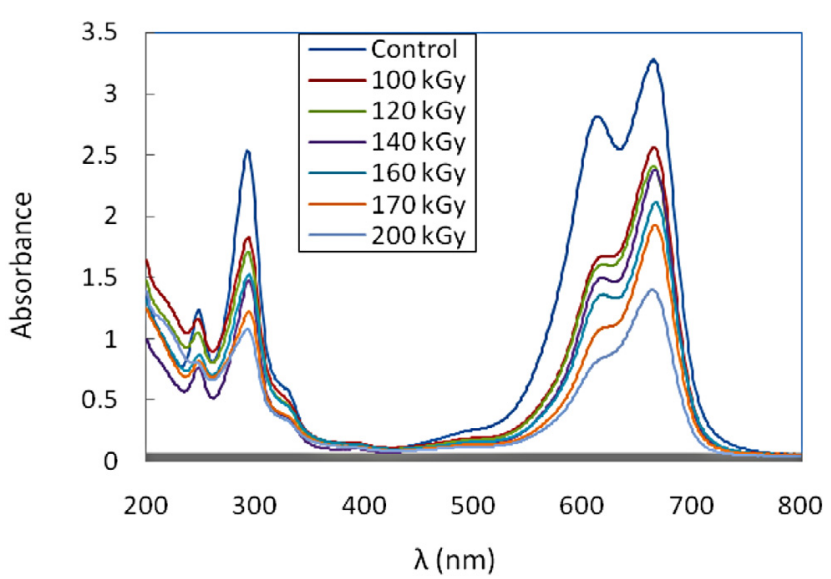

(b)

Figure 3. The absorption spectra of unirradiated and irradiated films with $0.4 \mathrm{~g} \mathrm{~L}^{-1}$ dye concentration. The films were irradiated with different ${ }^{137} \mathrm{Cs} \gamma$-ray doses from 100-200 kGy: (a) MR-PVA films, (b) MB-PVA films.

acceptable agreement with the earlier experimental results for investigating the optical absorbance. In order to obtain effective results of the PVA films with methyl red and methylene blue dyes by using the UV/VIS spectrophotometer, we had to consider different factors to be chosen for the implementation of the experiment. The system runtime (total length), humidity, temperature during irradiation and $\mathrm{pH}$ of the solution can be varied to study how efficiently the absorbance spectra of the polymer films compute the optical absorbance at $\lambda_{\max }$.

\subsection{Absorption spectra}

To examine the dosimetric characteristics, dyed and undyed films were exposed to a dose range from 100 to $200 \mathrm{kGy}$ and their absorption spectra were recorded using a UV/VIS spectrophotometer, as shown in Figure 2. The absorbance of undyed films was increased by increasing the dose with an absorbance peak at $272 \mathrm{~nm}$ (Chung et al., 1994). It is significant that our new investigation of the undyed PVA film generates pale brownish coloration at high doses (>150 kGy)

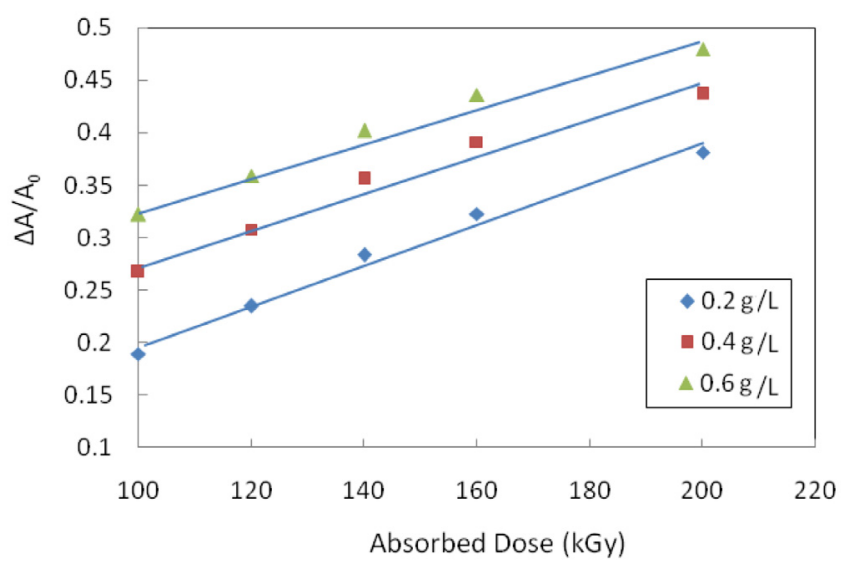

(a)

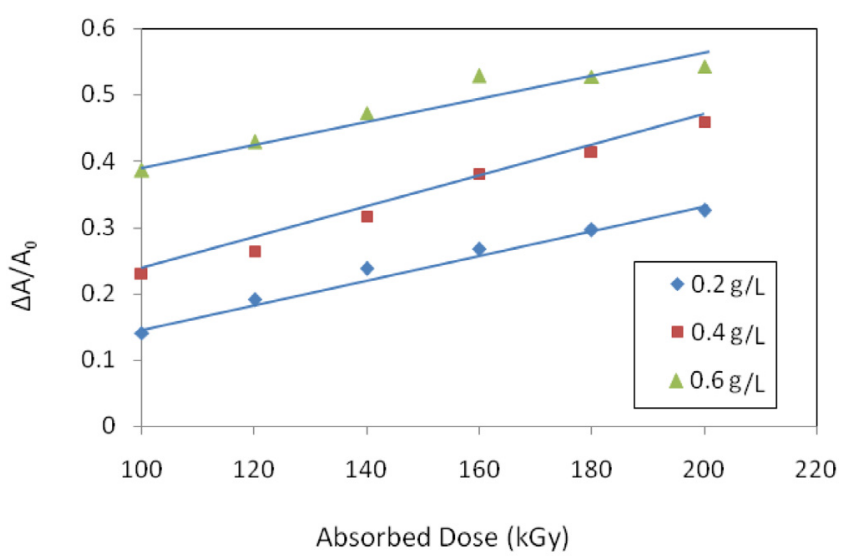

(b)

Figure 4. Dose-response curves of the films containing different dye concentrations $\left(0.2 \mathrm{~g} \mathrm{~L}^{-1}, 0.4 \mathrm{~g} \mathrm{~L}^{-1}\right.$ and $\left.0.6 \mathrm{~g} \mathrm{~L}^{-1}\right)$ upon $\gamma$-irradiation at peak wavelengths $\left(\lambda_{\max }\right)$ : (a) MR-PVA films at $\lambda_{\max }=488 \mathrm{~nm}$, (b) MB-PVA films at $\lambda_{\max }=668 \mathrm{~nm}$.

due to the formation of the double bonds in the chain (Kattan et al., 2011); therefore, it can be used as a dosimeter.

The absorption spectrum of the dyed PVA film for a dose range of 100-200 kGy of MR-PVA and MB-PVA films containing dye concentrations exposed to different doses of gamma rays was investigated. The absorption spectra of the MR-PVA and MB-PVA films were in the visible region with maximum peaks located at $518 \mathrm{~nm}$ and $668 \mathrm{~nm}$, respectively, with a shoulder peak of the MB-PVA film at $616 \mathrm{~nm}$, which agrees with the published results. (Chung et al., 1994).

Figure 3 illustrates that the amplitude of both (MR-PVA and MB-PVA films) absorption bands increases gradually with increasing dose without a change in positions at the same peak wavelength. It is also observed from Figure 3 that the change in color intensity of the films varied with the radiation dose. Free radicals are produced from PVA and induce a reduction reaction with azo-groups, resulting in the disappearance of the chromophore (Bhat et al., 2007; Alzahrany et al., 2011). The MR-PVA film experienced a change in color from red to colorless up to a dose of $200 \mathrm{kGy}$, while the MB-PVA film changed from dark blue to light blue. 


\subsection{Effect of dye concentration on the performance of the films}

The effect of the dye concentration on the response of the films was investigated by a relative absorbance $\left(\triangle \mathrm{A} / \mathrm{A}_{0}\right)$ relation at the peak wavelengths versus the absorbed dose (AbdelFattah et al., 1999) as shown in Figure 4, where $\Delta \mathrm{A}$ is the change in absorbance and $\mathrm{A}_{0}$ is the absorbance before irradiation. $\Delta \mathrm{A}=\mathrm{A}_{0}-\mathrm{A}_{i}$ and $\mathrm{A}_{0}$ and $\mathrm{A}_{i}$ are the values of optical absorbance at the peak wavelengths of unirradiated and irradiated films, respectively.

Abdel-Fattah and El-Kelany (1998) showed that the sensitivities are mainly dependent on the optical absorbance of the unirradiated films. Moreover, the optical absorbance of the unirradiated films $\left(\mathrm{A}_{0}\right)$ depends on the concentration and temperature of the solution during the synthesis of the films. Due to this reason, the dose response behaviors of the MR-PVA and MB-PVA films are established in terms of the change in absorbance divided by the absorbance before irradiation $\left(\triangle \mathrm{A} / \mathrm{A}_{0}\right)$ at the peak wavelengths as a function of the absorbed dose, where in order to investigate the effect of the dye concentration on the performance of the film dosimeters, the three sets of films for different concentrations of MR and MB dyes were prepared separately and exposed to high $\gamma$-radiation: (100200) kGy. The dose response curves for the MR-PVA and MBPVA films are shown in Figures $4 a$ and $4 b$, respectively. It is evident from Figure 4 that the relative absorbance varied linearly with doses up to $160 \mathrm{kGy}$, and the relative absorbance of the films was increased with the increase in the dye concentration. This result indicates that the films with higher dye concentration are more suitable for higher-dose film dosimetry. This is in good agreement with previous work on different types of dye (Alzahrany et al., 2011; Basfar et al., 2012).

A linear regression was performed for the MR-PVA and MB-PVA films, and lines are represented by the following relationships: $\mathrm{D}=403.6\left(\triangle \mathrm{A} / \mathrm{A}_{0}\right)_{518}$ for the MR-PVA film and $\mathrm{D}=438.66\left(\triangle \mathrm{A} / \mathrm{A}_{0}\right)_{668}$ for the MB-PVA film, where $\mathrm{D}$ is the absorbed dose in $\mathrm{kGy},\left(\triangle \mathrm{A} / \mathrm{A}_{0}\right)_{518}$ is the change in absorbance divided by the absorbance before irradiation at $518 \mathrm{~nm}$ wavelength for the methyl red film and $\left(\triangle \mathrm{A} / \mathrm{A}_{0}\right)_{668}$ is the change in absorbance divided by the absorbance before irradiation at $668 \mathrm{~nm}$ wavelength for the methylene blue film. The above relations can be used for the evaluation of the absorbed dose corresponding to a radiation-induced decrease in absorbance of the MR-PVA and MB-PVA films.

\subsection{Stability of the films before and after irradiation}

In order to study the stability of the dyed PVA films before and after irradiation, the films of $0.4 \mathrm{~g} \mathrm{~L}^{-1}$ dye concentration were stored in the dark and under laboratory fluorescent light at room temperature for 30 days. By measuring the absorbance of the unirradiated MR-PVA film after 30 days, it was noted that its absorbance at $518 \mathrm{~nm}$ remained unchanged under both storage conditions. Moreover, the irradiated MR-PVA films showed good stability in darkness and satisfactory stability in florescent light, with less than 3\% decrease in the absorbance. However, in the case of the unirradiated MB-PVA film, its absorbance at $668 \mathrm{~nm}$ was slightly changed after 30 days under both storage conditions. It is also observed that by irradiating the MB-PVA film, it regains its color after a few days in darkness and fluorescent light. It was found by Chung and Miller (1994) that most of the decolorization can be reversed upon admission of oxygen.

It is concluded from Figure $2 b$ that the MB-PVA film is not completely bleached up to $200 \mathrm{kGy}$. It is shown that approximately $65 \%$ of the MB-PVA film was bleached at $200 \mathrm{kGy}$ of the unirradiated film (absorbance $=3.3$ at $0 \mathrm{kGy}$ ), while in the earlier results of Chung et al. (1994), methylene blue PVA film was almost completely bleached (approximately 90\%) at $105 \mathrm{kGy}$ of the unirradiated MB-PVA film. We can suggest two possible reasons for this difference in the MB-PVA film bleaching. First, in the current experiment, a ${ }^{137} \mathrm{Cs}$ gamma source (energy $662 \mathrm{keV}$ ) is used for irradiation of the PVA films instead of a Co-60 gamma source (energy $1.17 \mathrm{MeV}$ or $1.33 \mathrm{MeV}$ ), which was used in the earlier results. Second, the concentration value used for methylene blue $\left(0.4 \mathrm{~g} \mathrm{~L}^{-1}\right)$ in the present film is very high compared with the value reported in the previous experiment (Chung et al., 1994), where the value of the concentration in the film was $0.04 \mathrm{~g} \mathrm{~L}^{-1}$ for methylene blue. It is expected that due to this reason, the useful dose range for methylene blue film at a high concentration has been extended, which is used for a wide range of applications in radiation processing, but in contrast the useful dose range noted in the previous work did not exceed $105 \mathrm{kGy}$, which was particularly used only for dose mapping. Moreover, the high concentration of methyl red indicator used in the MR-PVA film also improves the useful dose range for radiation processing. It is noted that approximately $85 \%$ of the methyl red film was bleached at $200 \mathrm{kGy}$ of the unirradiated film because MR indicator is an azo-dye, which has a carboxyl group in an orthoposition to the phenyl ring of the molecule. A change in the chemical structure of the MR molecule (for instance, the split of the carboxyl group due to gamma irradiation or interruption in the conjugated system or breakage of the azo group by gamma irradiation) can cause a bleaching of the color of the indicator (Ajji, 2006).

\section{Conclusions}

Radiochromic films were developed by using polyvinyl alcohol (PVA) with different dye concentrations (MR and MB: $0.2 \mathrm{~g} \mathrm{~L}^{-1}, 0.4 \mathrm{~g} \mathrm{~L}^{-1}$ and $\left.0.6 \mathrm{~g} \mathrm{~L}^{-1}\right)$ exposed at high doses of $\gamma$ irradiation: (100-200) kGy. We analyzed these films by using a UV/VIS spectrophotometer. Our investigations showed that the MR-PVA and MB-PVA films have a main absorption peak at wavelengths of $518 \mathrm{~nm}$ and $668 \mathrm{~nm}$, respectively, and the amplitude of both absorbance bands increases gradually with an increase in the absorbed dose. It was shown that the change in color intensity of both films was proportional to the dose, from red to colorless for the MR-PVA film, and from dark blue to light blue for the MB-PVA film. The dose response for the MR-PVA and MB-PVA films is linear for the range of $(100-160) \mathrm{kGy}$. The relative absorbance $\left(\triangle \mathrm{A} / \mathrm{A}_{0}\right)$ of both films increases significantly with an increase in the dye concentration in the films. Comparison of the results obtained with the films (MR-PVA and MB-PVA), different dye concentrations and different high doses of $\gamma$-irradiation strengths with earlier 
experimental results generally predicted well that the MR-PVA film has good stability in darkness and florescence light and the MB-PVA film also has satisfactory stability in darkness but is strongly affected by light. The efficiency and simplicity of the films are strong advantages; they are easy to prepare and allow fast repeated experiments on small systems, making the investigations very effective. The UV/VIS spectrophotometer is a powerful tool for analyzing and exploring the behavior of radiochromic films over different parameters with new extended polyvinyl alcohol dye films.

\section{References}

Abdel-Fattah A.A., El-Kelany M. (1998) Radiation-sensitive indicator based on radiation-chemical formation of acids in polyvinyl butyral films containing chloral hydrate, Radiat. Phys. Chem. 51 , 317-325.

Abdel-Fattah A.A., Said F.I.A., Ebraheem S., El-Kelany M., El Miligy A.A. (1999) Dyed acrylic-acid grafted polypropylene films for high-dose radiation dosimetry, Radiat. Phys. Chem. 54, 271-277.

Ajji Z. (2006) Usability of aqueous solutions of methyl red as highdose dosimeter for gamma radiation, Radiat. Meas. 41, 438-442.

Al Zahrany A.A., Rabaeh K.A., Basfar A.A. (2011) Radiationinduced color bleaching of methyl red in polyvinyl butyral film dosimeter, Radiat. Phys. Chem. 80, 1263-1267.
Basfar A.A., Rabaeh K.A., Mousa A.A. (2012) Improved performance of nitro-blue tetrazolium polyvinyl butyral high dose film dosimeters, Radiat. Meas. 47, 1005-1008.

Beshir W.B. (2013) Radiation sensitive indicator based on tetrabromo phenol blue dyed poly(vinylalcohol), Radiat. Phys. Chem. 86, 129-135.

Bhat N.V., Nate M.M., Bhat R.M., Bhatt B.C. (2007) Effect of $\gamma$ irradiation on polyvinyl alcohol films doped with some dyes and their use in dosimetric studies, Indian J. Pure Appl. Phys. 45, 545-548.

Chung W.H., Miller A. (1994) Film dosimeters based on Methylene Blue and Methyl Orange in Polyvinyl Alcohol, Nucl. Technol. 106, 261-264.

Kattan M., Kassiri H., Daher Y. (2011) Using polyvinyl chloride dyed with bromocresol purple in radiation dosimetry, Appl. Radiat. Isotopes 69, 377-380.

Lavalle M., Corda U., Fuochi P.G., Caminati S., Venturi M., Kovacs A., Baranyai M., Safrany A., Miller A. (2007) Radiochromic film containing methyl viologen for radiation dosimetry, Radiat. Phys. Chem. 76, 1502-1506.

Rabaeh K.A., Basfar A.A., Moussa A.A. (2012) Enhancement in sensitivity of nitro blue tetrazolium polyvinyl alcohol film dosimeters by sodium formate and Triton X-100, Radiat. Phys. Chem. 81, 479-483.

Susilawati Doyan A. (2009) Dose Response and Optical Properties of Dyed Poly Vinyl Alcohol-Trichloroacetic Acid Polymeric Blends Irradiated with Gamma-Rays, Am. J. Appl. Sci. 6, 2071-2077.

Cite this article as: S. Akhtar, A. Shahzad, S. Bashir, M.Y. Hussain, N. Akhtar. Improved performance of radiochromic films for high-dose dosimetry. Radioprotection 51(2), 129-133 (2016). 